\title{
Performance analysis of beam divergence propagation through rainwater and snow pack in free space optical communication
}

\author{
Mustafa H. Ali ${ }^{1}$, Rehab I. Ajel ${ }^{2}$, Samira Abdul-Kader Hussain ${ }^{3}$ \\ ${ }^{1}$ Department of Basic Sciences, College of Dentistry, Mustansiriyah University, Baghdad, Iraq \\ ${ }^{2,3}$ Department of Computer Science, College of Science, Mustansiriyah University, Baghdad, Iraq
}

\begin{tabular}{l} 
Article Info \\
\hline Article history: \\
Received Oct 8, 2020 \\
Revised Dec 4, 2020 \\
Accepted Apr 1, 2021 \\
\hline Keywords: \\
Beam divergence \\
Carrier-suppressed return-to- \\
zero (CSRZ) \\
Free space optics (FSO) \\
Rain \\
Snow
\end{tabular}

\begin{abstract}
In the present work the future communication requirements need to fulfill with high data rate, FSO (free space optic) with it is tremendous potential is the solution. This research observed the effectiveness analysis of FSO systems by modifying one of the most important FSO parameters beam divergence, under the most affected weather attenuating condition Rainwater and snow pack. The simulation is obtained and analyzed under single channels CSRZ-FSO (carrier-suppressed return-to-zero/free space optical) systems having capacity of $40 \mathrm{Gbps}$ between two transceivers with variable distance. The connection is presently under 5 meteorological turbulences (light rain, medium rain, wet snow, heavy rain and dry snow). The results show the heavy rain and dry snow have a very high attenuation carried out in terms of Q-factor. this result led us to conclude that small divergence offers significant performance improvement for FSO link and this performance decrease every time the beam divergence increase, Therefore, to build inexpensive and reliable transmission media, we go with new method that still in the experiment area called hybrid RF/FSO (radio frequency/free space optical) that compatible with atmospherically status.
\end{abstract}

This is an open access article under the CC BY-SA license.

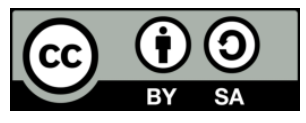

\section{Corresponding Author:}

Mustafa H. Ali

Department of Basic Sciences

College of Dentistry, Mustansiriyah University

Baghdad, Iraq

Email: mustafa.h@uomustansiriyah.edu.iq

\section{INTRODUCTION}

The need for advanced bandwidth is growing these days, according to the many applications that required a very high data rate communication, older wireless communication technology which connect remote sites like RF (radio frequency) doesn't satisfy the market in the near future, therefore the need of switching our communication system from the RF domain to optical domain is necessary [1]. Thus, FSO technology presented that considered promising technology that can meet the very high speed and has the capability of providing a LOS (line of site) wireless link with very high bandwidths. FSO in the first place was developed by the NASA (National Aeronautics and Space Administration) and military. It is important for a variety of communication applications float to surface since three decades ago [2], [3]. Modern improvement in FSO technology has increased for short, medium and long range communication applications as shown in Figure 1. FSO present specially to solve last mile access bottleneck problem. It also can be found in many other applications like high speed interplanetary internet links, real time medical imaging transfer, enterprise networking, work-sharing capabilities, video teleconferencing, video on demand, and video, streaming audio, data library access, electronic commerce and delay free web browsing [4], [5]. 


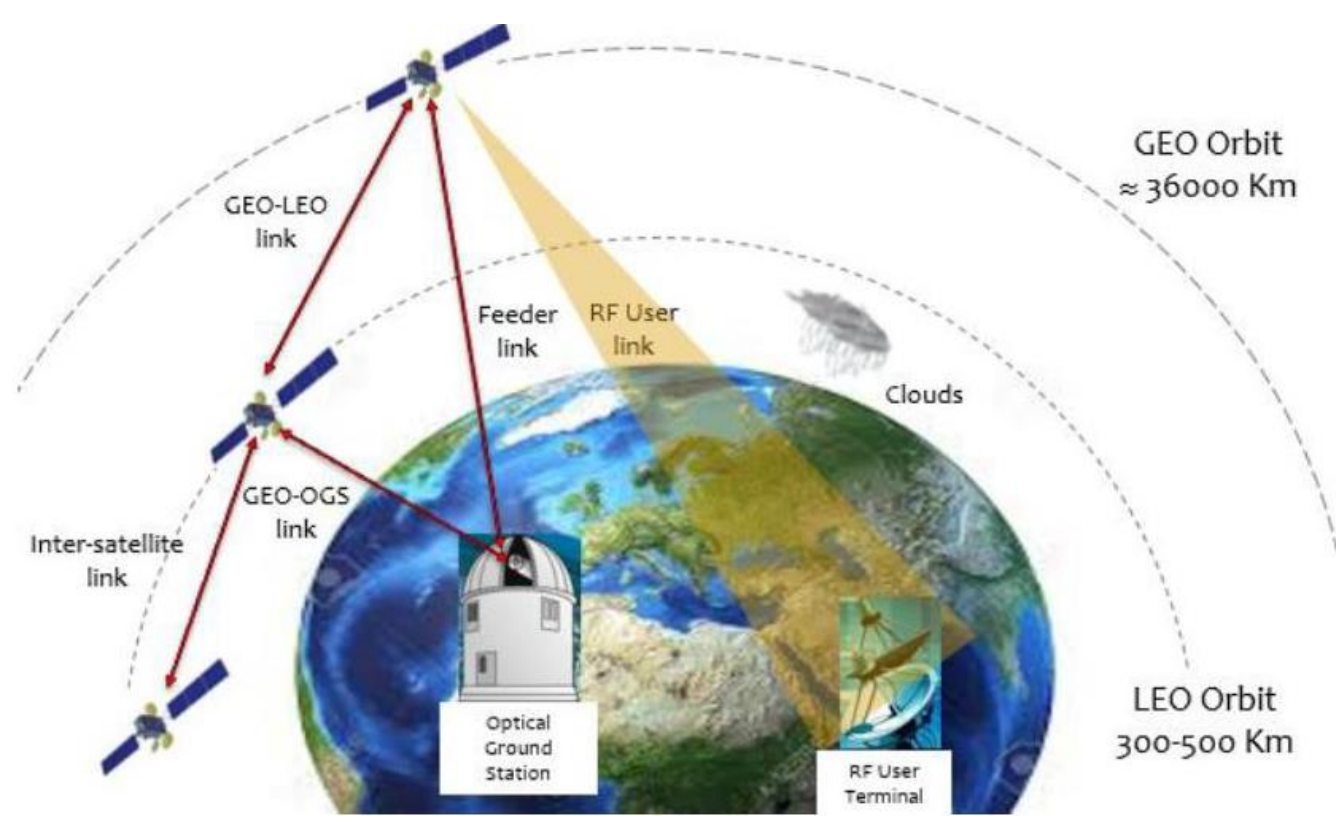

Figure 1. Free space optical network including both of LEO (low earth orbit) and GEO (geosynchronous earth orbit) scenarios [6]

FSO has many advantages like:

- Optical system spectrum has license free till now, this minimizes the development time and initial set up cost [7].

- FSO communication system has high security because of it is strongly directional beam laser with narrow beam divergence make it a very difficult for any kind of interception and thus cannot be detected by RF meters or spectrum analyzers. In addition, FSO signal does not have the ability to penetrate walls, thus make it prevent eavesdropping.

- Duo to compact and lightweight, FSO techniques component is easily installed, deploy and expandable in the future.

- Cover a variety geographic area where optic cables cannot be used.

- Simple and low cost because digging up the ground required a large effort in addition to laying down the new optical fiber, therefore the fiber optic has been pointed towards connecting the central offices with optical fiber backbone, and left the connecting of houses to central offices in share of FSO.

- High bandwidth and low bit error rate compared to the RF were affected by electromagnetic interference, in addition it has a technology minimizes the topology of network parts [8].

FSO communication system has its own disadvantage which they are:

- Because of the narrow beam divergence, it is hard to provide the requirement tracking and pointing of ATP (adenosine triphosphate) system and tight acquisition [9].

- FSO dependent on meteorological conditions which minimize the performance of the system and cannot be predictable [10].

- The system performance may decrease in some specific transmitter and receiver alignment of the position of the laser with the solar background radiations.

- Fibers optical can transfer a laser beam for huge distances while FSO is limited to shorter ranges.

In addition to the mention disadvantage, the major demolition problem that degrades the FSO link performance is to conquer different challenges produce by the heterogeneous nature of atmospheric channel [11]. Various atmospheric phenomena like adverse weather conditions, scintillation of the connecting signal, atmospheric turbulence, diffusion, scattering and absorption through it is impacting on FSO system and make vulnerable as shown in Figure 2. Several recent studies in Ahmedabad [12], Monte Carlo [13], and other many cities [14]-[17] using FSO-SISO (single input-single output) and WDM (wavelength division multiplaxing)-FSO systems. In addition to that, using Manchester coded in [18] and PolSK (polarization shift keying) modulation in [19]. All these systems show subdues the effects of increase in beam divergence, attenuation factor, and further extends the communication range. 


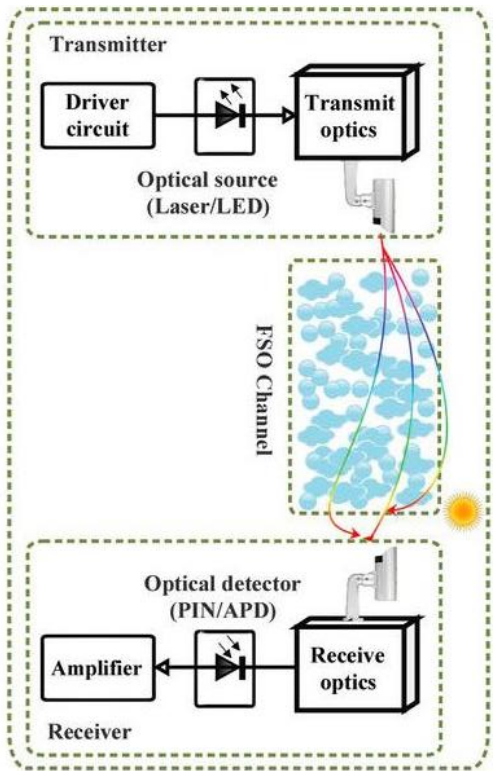

Figure 2. Block diagram of a terrestrial OWC (optical wireless communication) system [9]

Another recent study [20] of a BPolSK-(binary polarization shifts keying optical code division multiple access) it is found that system performance is highly dependent on transmission length. While Farhan et al. [21] using chaos and Barua and Majumder [22] using LDPC (low-density parity-check code) FSO communication employing OOK (on-off keying), BPSK (binary phase-shift keying), and QPPM (Q-ary pulse position) as modulation technique It is found that LDPC coded FSO system with QPPM provides significant coding gain over uncoded system compare than other modulation techniques. On another hand an OFDM (orthogonal frequency-division multiplexing) FSO link with optical intensity modulation and coherent homodyne receiver taking into consideration [23], [24]. It is noticed that effect of turbulence can be significantly reduced by increasing the number of OFDM subcarrier. Finally, Baiwa and Verma [25] shows different modulation formats (CSRZ, MDRZ (modified duobinary return to zero) and DRZ (duobinary return to zero) that conclude the CSRZ has the best performance in terms of Q factor and BER (bite errore rate) for rain and fog weather conditions. And that the reason we used CSRZ to analyses three different condition of rain and two different condition of snow.

The first part of the paper was simple background of the FSO and what tremendous potential can offer. In section two, the proposed method of the CSRZ in details is presented. In section three, a Simulation is introducing with the materials that build the design. Then, in section four, the various results that shows up from the design of the system were demonstrated under variables distance and weather condition in the influence of beam divergence effect which discussed later in the the fifth section and finally the sixth section shows the analysis conclusions from these variables.

\section{THE PROPOSED METHOD}

Terrestrial-FSO include communication between two ground terminal or between any type of horizontal connect. These FSO networks diffuse in their topology like P2P (point to point), P2MP (point to multipoint), mesh or ring as shown in Figure 3. Many factors affect the laser beam propagates through the atmosphere and led to power loss. Rain and snow are one of these factors and we will take them as an atmospheric problem. In this paper, we have transmitted 40 Gbps data through an FSO link by using CSRZ under the effect of different type of rain and snow, the performance of the proposed system is evaluated and analysis using a beam divergence angle.

The beam divergence is relative to $\lambda\left(D_{-}\right.$R) which $D R$ represent aperture diameter and $\lambda$ is the carrier wavelength. Consequently, comparing to the RF carrier, the beam diffusion presented by the optical carrier is narrower. This makes the transmitted power at the receiver side have growth in the signal intensity. Figure 4 shows a simple comparison for RF and optical beam divergence when a signal transmits from Mars back to Earth.

$1 \operatorname{mrad} \approx 0.0573^{\circ} \rightarrow 1^{\circ} \approx 17 \operatorname{mrad}$ 
$\theta$ is a FSO divergence angle among receiver and transmitter. The geometrical course loss for an FSO connection relies on the divergence angle $(\theta)$, the path length $(\mathrm{L})$, and optical transmitter beam-width. Receiver and Transmitter aperture diameters are a parameter that can be calculated and are commonly given by manufacturer. Spot size, angle and Small divergence angles among receiver and transmitter are shown in Figure 5.

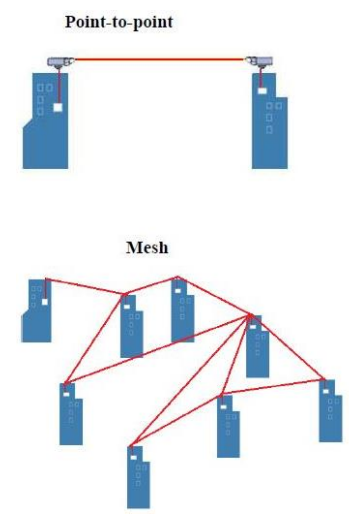

Figure 3. FSO network diffuse with P2P, P2MP, mesh or ring topology
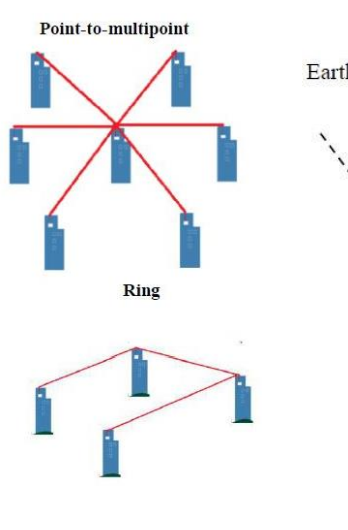

Figure 4. Beam divergence signal comparison of RF and optical

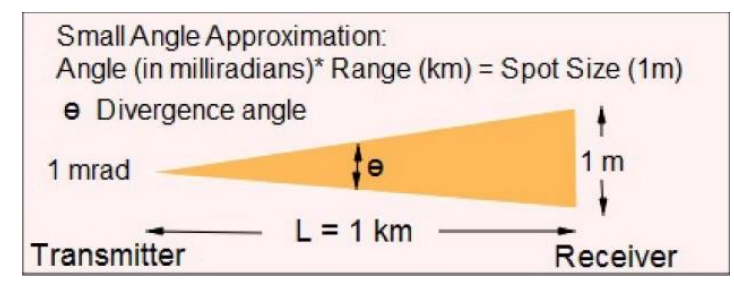

Figure 5. Spot size angle and small divergence angles among receiver and transmitter

\subsection{Rain}

The atmosphere contained water vapor that creates rain. It consists of water droplets that number and form are changing in space and time. Stroke law is used to calculate scattering coefficient of the rain as shown in (1):

$$
\beta_{\text {rain scat }}=\Pi a^{2} N_{a} Q_{\text {scat }}\left(\frac{a}{\lambda}\right)
$$

where: $\lambda$ : wavelength, $\mathrm{Na}$ : the rain drop distribution, $a$ : the radius of raindrop $(\mathrm{cm})$, and radius of rain drops $(0.001-0.1) \mathrm{cm}$. and $\mathrm{Q}_{\text {scat }}$ : the scattering efficiency. Intensity factors could affect the FSO performance and reduce the visibility. Therefore, the scattering coefficient as shown in (2) can be calculated using stroke law.

$$
\alpha_{\text {rain scat }}=\Pi a^{2} N a Q_{\text {scat }}\left(\frac{a}{\lambda}\right)
$$

Rain fall is describing by distribution in time, amount and intensity using (3) so we can calculate Na.

$$
N a=\left(\frac{R}{4 / 3\left(\Pi a^{3}\right) V_{a}}\right)
$$

where: $V_{a}$ : the limit speed precipitation and $R$ : the rainfall rate $(\mathrm{cm} / \mathrm{s})$. The raindrop distribution is also given in (4):

$$
v a=\left(\frac{2 a^{2} p g}{9 \eta}\right)
$$


where: $\eta$ : viscosity of air $1.8 \times 10^{-4}(\mathrm{~g} / \mathrm{cm}) \mathrm{s}, g=980 \mathrm{~cm} / \mathrm{sec}^{2} \cdot g$ : gravitational constant, $\rho$ : water density $(\rho=1$ $\left.\mathrm{g} / \mathrm{cm}^{3}\right)$. Attenuation of the rain can be calculated by utilizing Beer's law as shown in (5):

$$
\tau=\exp \left(-\beta_{\text {rain scat }} L\right)
$$

$\mathrm{s}$ the rainfall rate increases and raindrop sizes increase to be a few millimeters in diameter. The attenuation linearly increases. From these previous causes of attenuation, rain rate of $\mathrm{R} \mathrm{mm} / \mathrm{hr}$ of wireless optical link in $\mathrm{dB} / \mathrm{km}$ is given by (6):

$$
a_{\text {spec }}=1.076 R^{0.67}
$$

Although the clouds, dust particles and fog are the greatly disturbed the propagation of signals, the rain is still the superior attenuating element especially above $10 \mathrm{GHz}$.

\subsection{Snow}

Snow is a second serious attenuation factor observed in FSO. Beside the dependence on the visibility ranges, the attenuation depends on the proportional size of snow particles. Large snow molecules cannot ignorable, it causes connecting failure. It is varies as a function of temperatures and liquid content of snow are the main factors that density of snow strongly contingent too. As shown in (7)-(9), there are two classifications for snow "dry snow" and "wet snow". Generally, snow attenuation calculated by (7);

$$
a_{\text {snow }}=a \cdot S^{b}
$$

for dry snow a and $\mathrm{b}$ are as shown in (8);

$$
\begin{aligned}
& a=5.42 * 10^{-5} \lambda+5.4958776, \\
& b=1.38
\end{aligned}
$$

where $a=5.495$ and $b=1.38$ and for wet snow $a$ and $b$ are as are shown in (9);

$$
\begin{aligned}
& a=1.023 * 10^{-4} \lambda+3.7855466 \\
& \mathrm{~b}=0.72
\end{aligned}
$$

where, $a=3.78$ and $b=0.72$.

\section{SIMULATION}

Many mechanisms have been suggested to develop the effectiveness of an FSO link, for example as shown in Figure 6, CSRZ (carrier-suppressed return-to-zero) are offered as aloft spectral effectiveness, diversity, amplification of signal and aperture averaging, CSRZ is a shape of the RZ (return-to-zero) modulation format in with suppressed carrier.

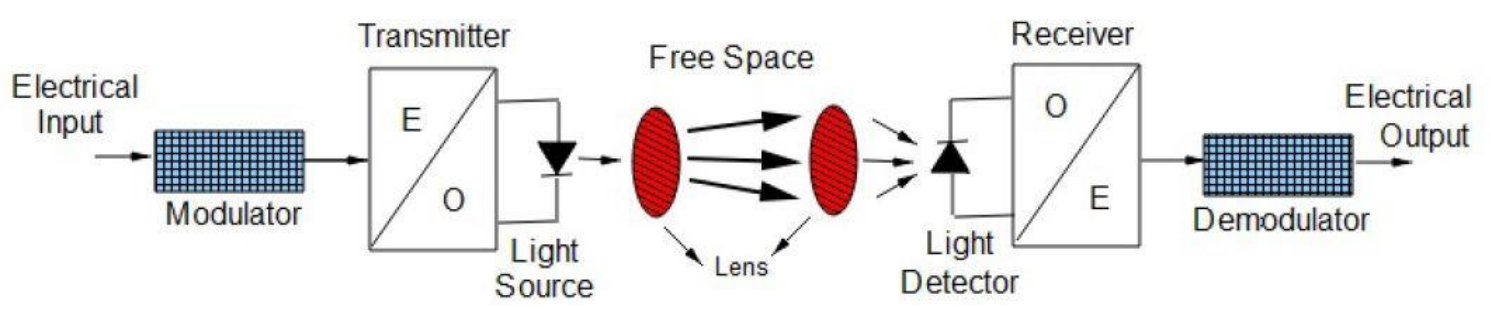

Figure 6. Block diagram explain the front end of a receiver and transmitter in optical wireless link

The CSRZ modulation format has many advantages over RZ modulation format duo to this better chromatic dispersion effect, group velocity dispersion and high tolerance to the self-phase modulation. Note that, because there is no DC (direct current) element for CSRZ, it displays minimal signal bandwidth comparing to traditional RZ. CSRZ has developed the spectral efficiency and the characteristics of diminishing the nonlinear impairments. 
Therefore, CSRZ is a represent the amount of divergence in the RZ in CSRZ the phase of the carrier is reversed for every symbol in RZ pulse modulated. By nonlinear effects CSRZ has more tolerance to degrade compared to NRZ (non-return-to-zero) and RZ and also has better transmission characteristics in a single channel. CSRZ results in minimizing inter symbol interference because of phase reversal among adjacent bits in the existence of various atmospheric effects as aforementioned, CSRZ have been inspected numerically to analysis their impacts in an FSO design with a single channel system as shown in Figure 7 shows the FSO simulation design system which include transmitter, atmosphere and receiver.

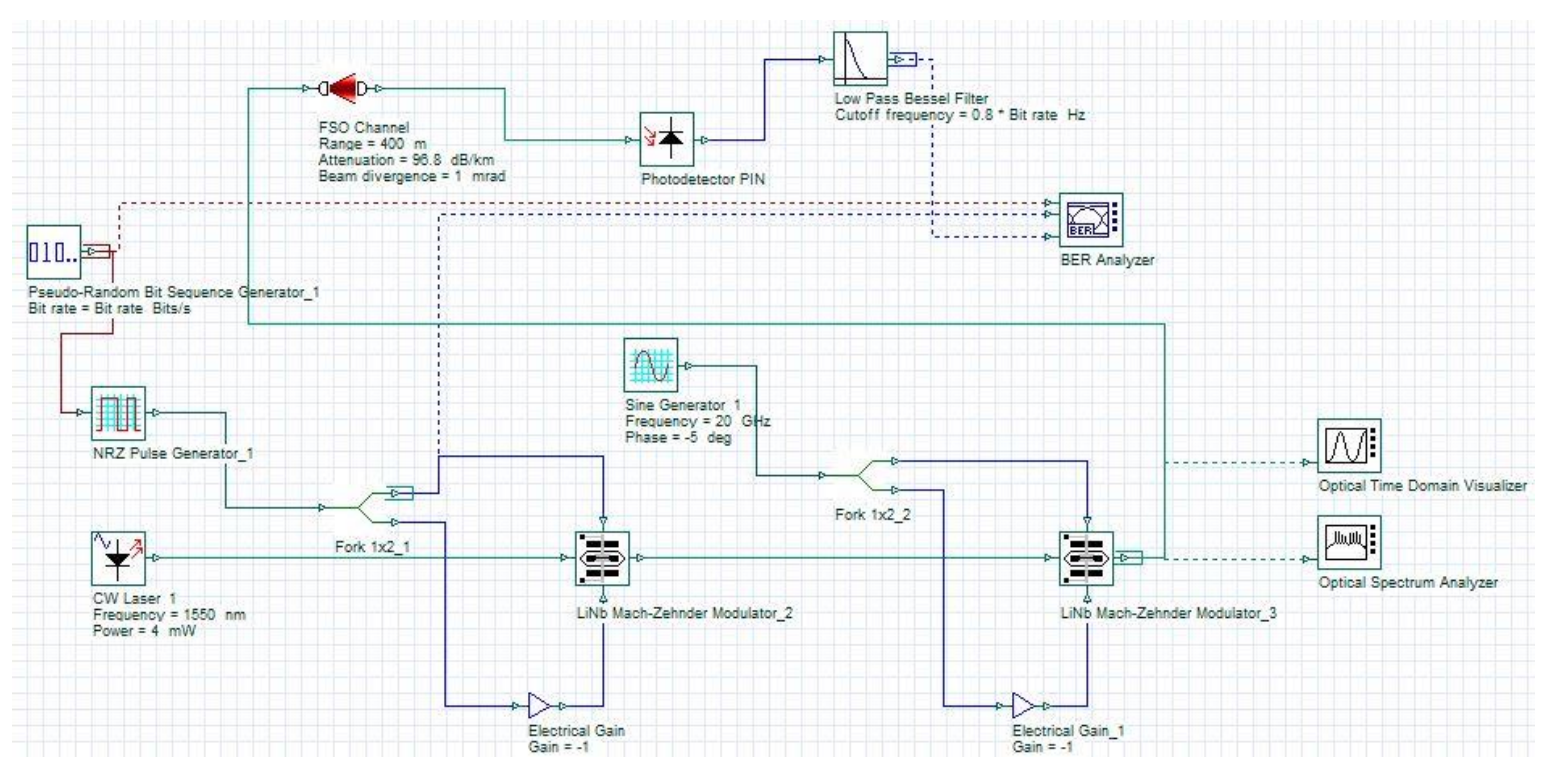

Figure 7. Block diagram of CSRZ modulation format

The transmitter includes Mach-Zehnder modulator, CW laser, modulation scheme driver and data source. The first subsystem is PRBS (pseudo random bit generator). It represents data transmission. The output of a PRBS is interred the NRZ pulse generators (to shape these pulses), then the output fed fork1, one of the fork output inter to electrical gain than to the Li-Nb MZ modulator_1 which represent the first input. The second input in $\mathrm{Nb} \mathrm{MZ \_ 1} \mathrm{is} \mathrm{from} \mathrm{the} \mathrm{continuous} \mathrm{wave} \mathrm{laser} \mathrm{(with} 4$ MW and $1550 \mathrm{NM}$ because of proliferation in the high quality detector and transmitter components and its low attenuation this wavelength band which make it eye safety and very well appropriate for FSO transmission) and finally the third input in $\mathrm{Nb} \mathrm{MZ} \_1$ from the fork1 directly. Then the output of $\mathrm{Nb} \mathrm{MZ \_ 1} \mathrm{becomes} \mathrm{first} \mathrm{input} \mathrm{to} \mathrm{a} \mathrm{Li}-\mathrm{Nb} \mathrm{MZ}$ modulator_2 that has also three inputs. A second input to Li-Nb MZ modulator_2 is directly from a sinusoidal that generator electrical signal with $20 \mathrm{GHz}$ with phase equal to $-90^{\circ}$ and third input also from the sinusoidal but after a pass through the electrical gain $(-1)$. Then via FSO channel sent to the PIN photo-detector in the receiver side that transforms the optical signal to an electrical signal. Then the output goes to a low pass bessel filter with a cutoff frequency equal to $0.75 \times$ bit rate of the signal to remove the unwanted high frequency, then from the BER analyzer we analyzed the Bit error pattern

\section{RESULT}

We present some advanced modulation techniques like as CSRZ to analysis the effectiveness evaluation of the FSO system under rain and snow weather conditions using the variable effect of beam divergence $(1,3,5,7$, and 10) Mrad to measure the significant role that the beam divergence played it in designing FSO links as atmospheric turbulences. Q-factor is drowning, against various distances for various attenuation forms that represent the 5 samples of weather as shown in Figures 8-12, which they are (light rain to medium rain then wet snow, heavy rain and dry snow). From the first look we notice that the value of Q-factor declines as the distance increases the main problem is with heavy rain and dry snow in the Figures 11 and 12 show the worst results among the others situation due to the diffraction close to receiver aperture and highly increased nonlinearities in another word beam divergence. This Geometrical loss in beam divergence came because Some part of the beam that transferred through the atmosphere will not be gathered 
by the receiver. This loss growth with the increase in the link extent, unless receiver diversity is employed or the dimension of the receiver aperture collection is raised.

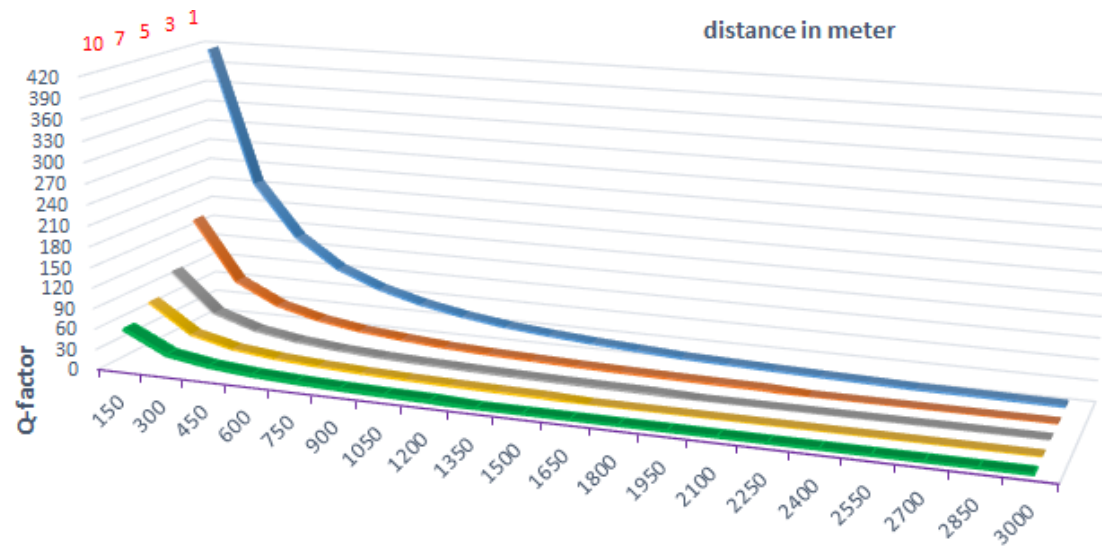

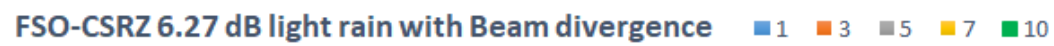

Figure 8. FSO-CSRZ $6.27 \mathrm{~dB}$ light rain with beam divergence

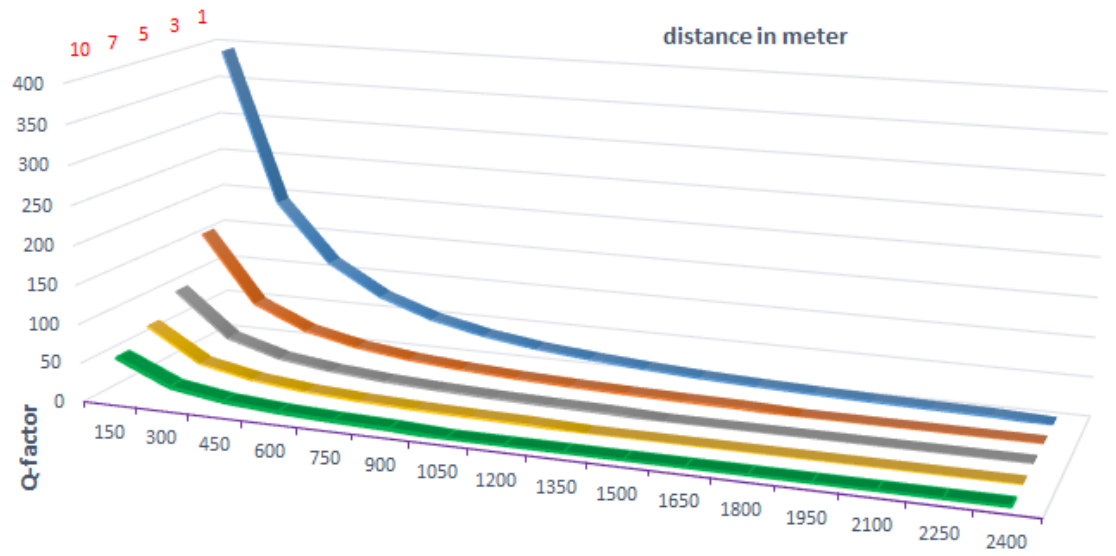

FSO-CSRZ 9.64 dB medium rain with Beam divergence $1=3 \backsim 5 \backsim 7 \backsim 10$

Figure 9. FSO-CSRZ 9.64 dB medium rain with beam divergence

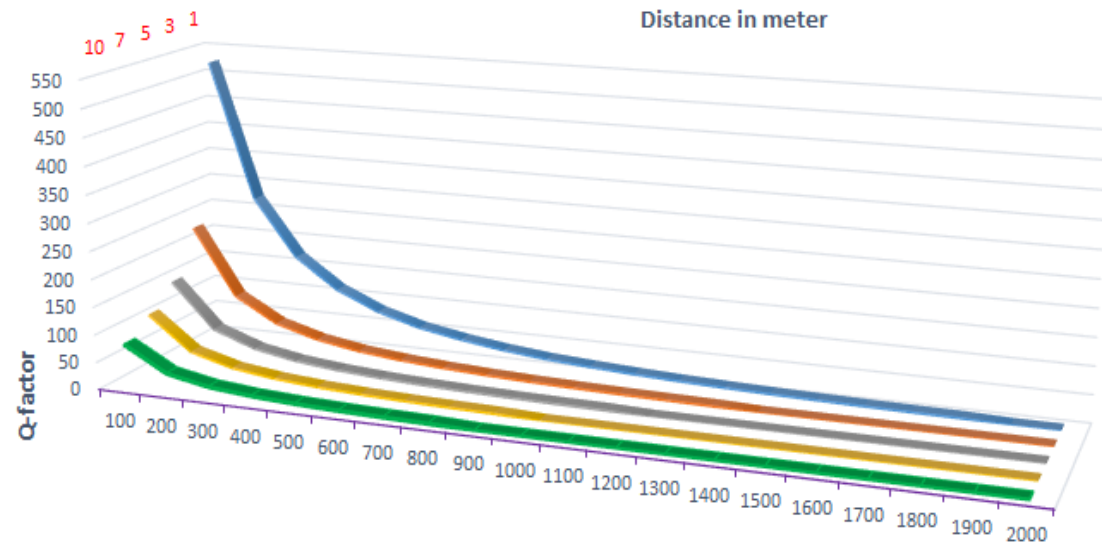

FSO-CSRZ 13.73 dB Wet snow with Beam divergence $\because 1=3=5 \backsim 7 \approx 10$

Figure 10. FSO-CSRZ $13.73 \mathrm{~dB}$ wet snow with beam divergence 


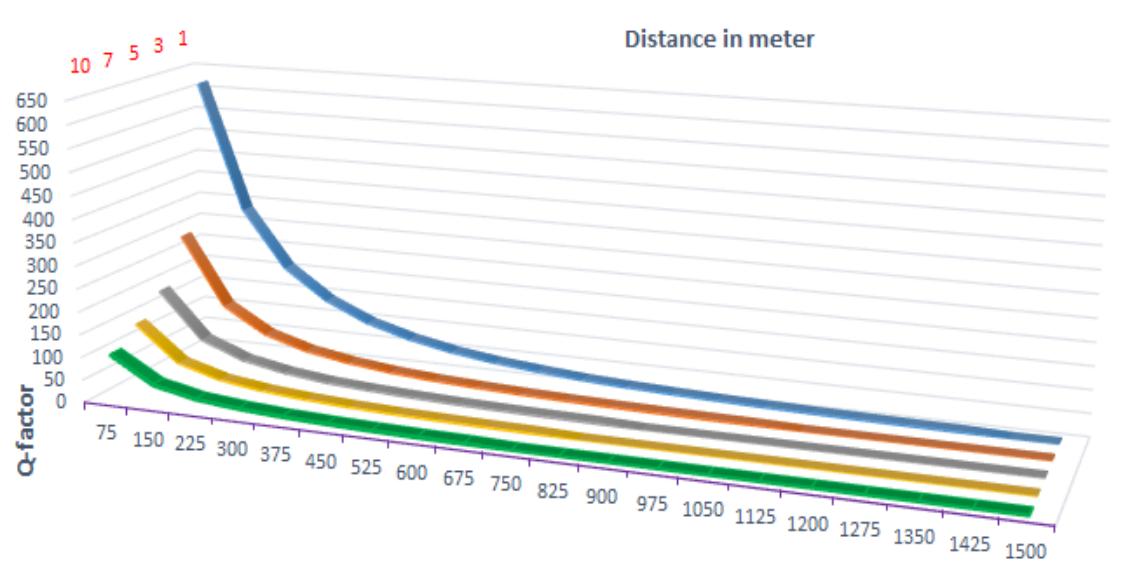

FSO-CSRZ 19.28 dB Heavy rain with Beam divergence $\square 1 \backsim 3 \backsim 5 \backsim 7 \backsim 10$

Figure 11. FSO-CSRZ 19.28 dB heavy rain with beam divergence

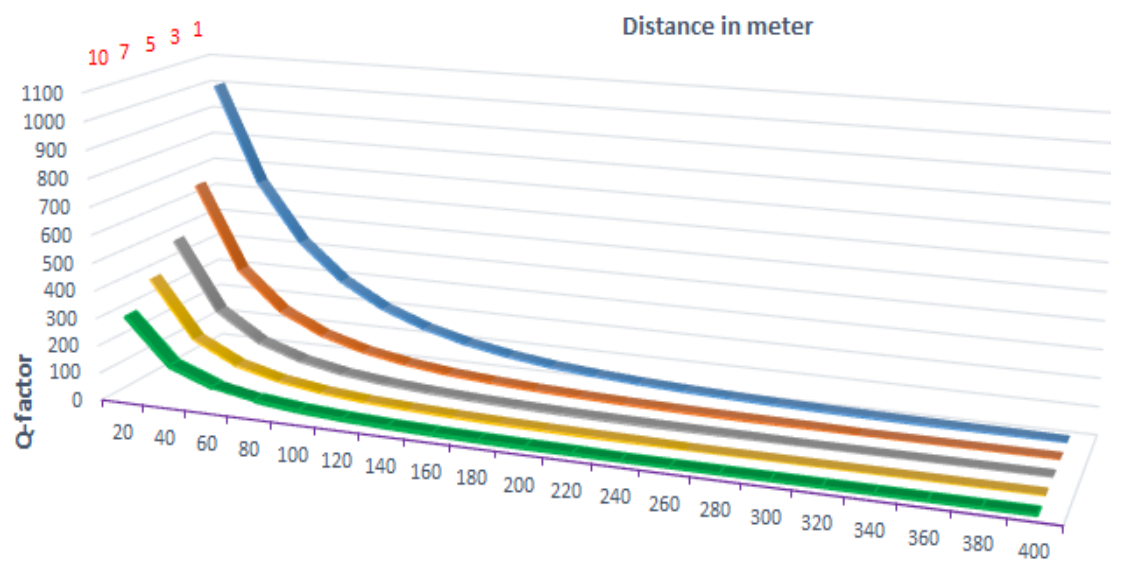

FSO-CSRZ 96.8 dB Dry snow with Beam divergence $₫ 1=3 \approx 5=7 \approx 10$

Figure 12. FSO-CSRZ $96.8 \mathrm{~dB}$ dry snow with beam divergence

\section{DISCUSSION}

Non-selective scattering in the phenomena that happened when FSO systems connect under rainfall, this is duo to the radius of raindrops equal to $100 \mu \mathrm{m}$ in light rain up to medium, then heavy rain with 1000 $\mu \mathrm{m}$ radius which is larger than the $1550 \mathrm{~nm}$ the wavelength of the laser in the standard FSO. As rain droplets are significantly larger as these droplets becomes quite large which led to reflection and refraction, consequently, generate the wavelength independent scattering, Therefore, the laser has the ability to go over the raindrop particle, having minimum scattering influence specially because it stays shorter in the atmosphere and the radius of the rain particles are significantly bigger. This is the major purpose that make attenuation through rain is minimum compared to haze (because haze stay longer in the atmosphere). And this makes the laser is more efficient the RF also because in the contrast, RF operate at frequencies above 10 $\mathrm{GHz}$ are closer match to the radius of raindrops in the other hand snow attenuation is split into wet and dry snow attenuation. In dry snow the laser beamis blocked because of the structure of ice on the window pane or due to increase density of snowflakes in the propagation path (slowly dropping), while wet snow particles has a diminution among heavy rain and fog particles make it fasterly dropping in the propagation path and led to block the laser beamis but not for long time, therefore, in both case led to huge attenuation (specially dry) and significantly minimize the connecting availability of FSO system.

\section{CONCLUSION}

It is obvious that after the huge progress in FSO communication system technology, there is still a many problems affect its performance and prevent it is growing prospects in the near future. the result led us 
to conclude that small divergence offers significant performance improvement for FSO link and this performance decrease every time the beam divergence increase. In addition, any connection with present of heavy rain or dry snow result a very high attenuation. We also don't forget the other important values, dependence upon rain drop size and rain temperature. Consequently, we didn't achieve inexpensive and reliable transmission media for huge data bandwidth using a single system RF or FSO and covered an acceptable distance. The recommendations are going with the development and research that present a hybrid $\mathrm{RF} / \mathrm{FSO}$ system for almost all weather conditions duo to: (i) It is ensuring carrier class availability, improving particularly for systems that operate at frequency equal to $10 \mathrm{GHz}$ and above; (ii) Improves the reliability of FSO systems by providing suitable protocols and algorithms in the upper model of TCP (transmission code protocol) layers like transport, and application; (iii) Capability to atmospherically status, which insert errors correction and can create the system usable for few times-periods.

\section{ACKNOWLEDGEMENTS}

The authors would like to thank Mustansiriyah University (www.uomustansiriyah.edu.iq) Baghdad-Iraq for it is support in the present work.

\section{REFERENCES}

[1] A. W. Y. Khang, S. J. Elias, J. Pusppanathan, N. Zulkifli, N. H. Halim, and S. Abdullah, "Last mile mobile hybrid optical wireless access network routing enhancement," Bulletin of Electrical Engineering and Informatics, vol. 8, no. 1, pp. 188-195, March 2019, doi: https://doi.org/10.11591/eei.v8i1.1434.

[2] M. Grover, P. Singh, P. Kaur, and C. Madhu, "Multibeam WDMFSO system: an optimum solution for clear and hazy weather conditions," Wireless Personal Communications, vol. 97, no. 5, pp. 5783-5795, 2017, doi: 10.1007/s11277-017-4810-2.

[3] N. Sahu and J. C. Prajapti, "Optimization of WDM-FSO link using multiple beams under different rain condition," Int. J. Adv. Res. Electron. Commun. Eng., vol. 4, no. 5, pp. 1125-1131, 2015.

[4] U. Darusalam, F. Y. Zulkifli, P. S. Priambodo, E. T. Rahardjo, "Hybrid optical communications for supporting the Palapa Ring network," Bulletin of Electrical Engineering and Informatics, vol. 9, no. 3, pp. 1055-1066, June 2020, doi: https://doi.org/10.11591/eei.v9i3.2008.

[5] F. U. Rashidi and S. K. Semakuwa, "Analysis of rain effect in free space optical communication under NRZ modulation in two regions," Int J Adv Eng Nano Technol (IJAENT), vol. 1, no. 10, pp. 13-16, 2014.

[6] J. Zhou, Y. Shao, Z. Wang, C. Li, Y. Zhou, and W. Ma, "A 16PSK-OFDM-FSO communication system under complex weather conditions" Optics and Photonics Journal, vol. 6, no. 8, pp. 131-135, 2016, doi: 10.4236/opj.2016.68B022.

[7] P. Kaur, V. K. Jain, and S. Kar, "Performance analysis of free space optical links using multi-input multi-output and aperture averaging in presence of turbulence and various weather conditions," IET Commun., vol. 9, no. 8, pp. 1104-1109, 2015, doi: 10.1049/iet-com.2014.0926.

[8] J-S. Lee, D-H. Lee, S-J. Kim, and C-H. Oh, "An LED-based visible light communication system for multicast," Indonesian Journal of Electrical Engineering and Computer Science, vol. 13, no. 1, pp. 265-271, 2019, doi: 10.11591/ijeecs.v13.i1.pp265-271.

[9] S. Robinson and S. Jasmine, "Performance analysis of hybrid WDMFSO system under various weather conditions," Frequenz, vol. 70, no. 9-10, pp. 433-41, 2016, doi: 10.1515/freq-2015-0287.

[10] K Thakur and A Sharma, "Comparison of MDRZ, CSRZ, and DRZ schemes using different communication channels," Int J Comput. Appl., vol. 172, no. 3, pp. 26-30, 2017, doi: 10.5120/ijca2017915106.

[11] B. Barua and S. P. Majumder, "Performance analysis of a multiple subcarrier modulated FSO communication system using direct detection optical receiver under the effect of weak atmospheric turbulence," Journal of Optical Communications, vol. 41, no. 4, pp. 453-461, Apr. 2020, doi: https://doi.org/10.1515/joc-2018-0006.

[12] D. Shah and D. K. Kothari, "Optimization of 2.5 Gbps WDM-FSO link range under different rain conditions in Ahmedabad," IEEE India Conference (INDICON), pp. 1-4, 2014, doi: 10.1109/INDICON.2014.7030643.

[13] M. Abazaa, R. Mesleh, and a. Mansour, "Performance analysis of MISO multi-hop FSO links over log-normal channels with fog and beam divergence attenuations," Optics Communications, vol. 334, pp. 247-252, Jan. 2015, doi: https://doi.org/10.1016/j.optcom.2014.08.050.

[14] G. Kar, P. Agarwalla, N. Roychoudhury, T. K. De, K. Pal, and S.Roy, "Analysis of a FSO link under different rain conditions," International Conference on Microwave and Photonics (ICMAP), IEEE, pp. 1-2, 2015, doi: 10.1109/ICMAP.2015.7408743.

[15] D. Kakati and S. C. Arya, "Performance of $120 \mathrm{Gbps}$ single channel coherent DP-16-QAM in terrestrial FSO link under different weather conditions," Optik, vol. 178, pp. 1230-1239, Feb. 2019, doi: https://doi.org/10.1016/j.ijleo.2018.10.035.

[16] T. N. Khajwal, A. Mushtaq, and S. Kaur, "Performance analysis of FSO-SISO and FSO-WDM systems under different atmospheric conditions," In 2020 7th International Conference on Signal Processing and Integrated Networks (SPIN), IEEE, pp. 312-316, 2020, doi: 10.1109/SPIN48934.2020.9071116.

[17] S. Chaudhary, P. Bansal, and M. Lumb, "Effect of beam divergence on WDM-FSO transmission system," International Journal of Computer Applications, vol. 93, no. 1, pp. 28-32, May 2014, doi: 10.5120/16181-5397. 
[18] M. Meena and S. K. Toshniwal, "Performance evaluation of manchester encoded input signal FSO system under different atmospheric condition for different wavelength," International Journal of Trend in Scientific Research and Development, vol. 3, no. 1, pp. 1331-1335, 2018, doi: 10.31142/ijtsrd21378.

[19] M. Rajeev, G. A. Mathew, and P. Krishnan, "Analysis of beam divergence on free space optical link using polarization shift keying technique," Optical Engineering, vol. 58, no. 4, p. 046109, Apr. 2019, doi: 10.1117/1.OE.58.4.046109.

[20] P. Kundu, Md. J. Islam, and I. J. Talukder, "Performance analysis of binary polarization shift keying OCDMA FSO system under different atmospheric conditions," In 2017 2nd International Conference on Electrical \& Electronic Engineering (ICEEE), IEEE, pp. 1-4, 2017, doi: 10.1109/CEEE.2017.8412911.

[21] A. Niaz, F. Qamar, M. Ali, R. Farhan, and M. K. Islam, "Performance analysis of chaotic FSO communication system under different weather conditions," Transactions on Emerging Telecommunications Technologies, vol. 30, no. 2, p. e3486, Aug. 2018, doi: 10.1002/ett.3486.

[22] B. Barua and S. P. Majumder, "Performance analysis of an LDPC coded FSO communication system with different modulation technique under turbulent condition," In 2012 15th International Conference on Computer and Information Technology (ICCIT), IEEE, pp. 240-243, 2012, doi: 10.1109/ICCITechn.2012.6509706.

[23] B. Barua and S. P. Majumder, "BER performance analysis of an orthogonal FDM free space optical communication system with homodyne optical receiver over turbulent atmospheric channel," Journal of Optical Communications(Ahead of print), pp 1-8, 2019, doi: 10.1515/joc-2019-0221.

[24] P. Patel, V. Mishra, and V. Singh, "Performance analysis of CO-OFDM FSO system under different weather conditions," In 2014 2nd International Conference on Emerging Technology Trends in Electronics, Communication and Networking, IEEE, pp. 1-5, 2014, doi: 10.1109/ET2ECN.2014.7044972.

[25] R. Baiwa and P. Verma, "Performance analysis of FSO system for advanced modulation formats under different weather conditions," In 2018 Second International Conference on Intelligent Computing and Control Systems (ICICCS), IEEE, pp. 1490-1495, 2018, doi: 10.1109/ICCONS.2018.8663090.

\section{BIOGRAPHIES OF AUTHORS}
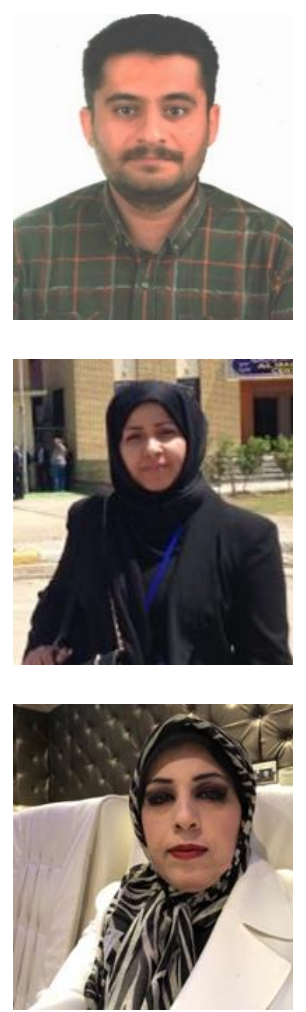

Mustafa H. Ali was born in Baghdad, Iraq, in 1990. He received the B.S. degree from Middle Technical University (MTU), Baghdad, Iraq in 2012, in specialty Computer Technical Engineering and the M.S. degree from Kazan State Technical University (named after A. N. Tupolev) Kazan, Russia in 2016, In specialty Info-communication technology and communication systems. He is currently working as a lecturer at Department of Basic Sciences /College of Dentistry/Mustansiriyah University. His research interests include Optical Communication Engineering, Passive Optical Network, Radio Over Fiber and Optical Wireless Communications.

Rehab I. Ajel was born in Baghdad, Iraq, in 1975. He received the M.S degree from Mustansiriyah University, Baghdad, Iraq in 2005. She is currently working as a lecturer at Department of Computer Science/College of Science/Mustansiriyah University. Her research interests include optical communication, neural network and genetic algorithms.

Samira Abdul-Kader Hussain was born in Baghdad, Iraq, in 1971. She received the M.S degree from University of Babylon, Babel, Iraq in 2012. She is currently working as a lecturer at Department of Computer Science/College of Science/Mustansiriyah University. Her research interests include satellite image processing, upgrade GIS and integrate GIS system with image processing. 